\title{
SURE-BASED MOTION BLUR ESTIMATION
}

\author{
Feng Xue and Thierry Blu
}

\section{Department of Electronic Engineering, The Chinese University of Hong Kong, Hong Kong}

\begin{abstract}
We propose a novel approach to estimate the parameters of motion blur (blur length and orientation) from an observed image.

The estimation of the motion blur parameters is based on a novel criterion - the minimization of an unbiased estimate of a filtered MSE ("blur-SURE"). By finding the best Wiener filter for this criterion, we automatically find the blur parameters with high accuracy. We then use these parameters in a recent (non-blind) deblurring algorithm that we have proposed and that achieves the state-of-the art in deconvolution.

The results obtained are quite competitive with other standard algorithms under various range of scenarios: high noise level, short blur length, etc.
\end{abstract}

Index Terms - motion blur, blur orientation, blur length, minimization of blur SURE, Wiener filtering

\section{INTRODUCTION}

Motion blur is one of the most common blurs in blind image deblurring problem, which has also been frequently encountered in real application, e.g. camera shake and sports photography $[1,2]$. Hence, it has attracted considerable attention in recent years. In this problem, image formation can be mathematically modeled as [3-5]:

$$
\mathbf{y}=\mathbf{H}_{0} \mathbf{x}+\mathbf{n}
$$

where $\mathbf{y} \in \mathbb{R}^{N}$ and $\mathbf{x} \in \mathbb{R}^{N}$ are the observed and original unknown images, respectively. $\mathbf{H}_{0} \in \mathbb{R}^{N \times N}$ is the convolution matrix constructed by the corresponding unknown true PSF (point spread function) $\mathbf{h}_{0}(u, v), \mathbf{n} \in \mathbb{R}^{N}$ is the additive Gaussian white noise with variance $\sigma^{2}$. Here, $N$ denotes the pixel number, $(u, v)$ is the 2-D Cartesian coordinates in the spatial domain.

The task of motion deblurring is to estimate the original image $\mathbf{x}$, knowing the observed data $\mathbf{y}$ only [2]. Note that the accurate estimation of PSF is of great importance to obtain a good estimate of $\mathbf{x}$ [6]. Several non-parametric methods have been proposed to estimate PSF, within Bayesian framework or regularization techniques, by imposing a certain prior knowledge on blur kernel $\mathbf{h}(u, v)[1,7]$.

This work was supported by a General Research Fund CUHK 410110 from the HK Research Grant Council.
If the motion blur is linearly constant, the convolution kernel $\mathbf{h}(u, v)$ can be expressed as $[3,4]$ :

$$
\mathbf{h}(u, v)= \begin{cases}\frac{1}{L} & \text { if } \sqrt{u^{2}+v^{2}} \leq \frac{L}{2} \text { and } \frac{u}{v}=-\tan \theta \\ 0 & \text { otherwise }\end{cases}
$$

with two key parameters: (1) blur length $L$ measured by the pixel number; (2) blur orientation $\theta$ evaluated by the angle with respect to the horizontal direction in degrees. Thus, the blur identification problem boils down to estimating the parameters: $L$ and $\theta$, from the observation $\mathbf{y}$ [3-6].

Recently, people have developed various methods to estimate the two parameters, which falls into the following three categories.

1. The cepstral method $[4,8]$ : after computing the 2-D cepstrum of the blurred image, there will be two pronounced negative peaks in the cepstrum. $L$ and $\theta$ are estimated by finding the two peak points.

2. Radon transform method $[5,9]$ : Radon transform takes integrals along different angles in the blurred image. It can be observed that the integral is maximized along the motion direction.

3. Steerable filters method $[3,10]$ : by applying a steerable filter to the power spectrum of the blurred image, the blur direction $\theta$ is estimated by detecting the maximum response value. It is less accurate than Radon transform.

Most of the existing methods need to analyze the spectrum of the blurred image to identify the motion parameters. Unlike them, we propose a new method to estimate blur length and orientation, based on the minimization of a modified SURE. Recently, SURE ${ }^{1}$ has shown to be a powerful tool for image denoising and deconvolution [11-13]. Here, we extend the SURE-based approach to estimate the parameters of motion blur, then, apply our proposed SURE-LET approach to perform non-blind decnovolution with the estimated blur kernel [14].

\section{PSF ESTIMATION BASED ON THE MINIMIZATION OF BLUR SURE}

\footnotetext{
${ }^{1}$ SURE: acronym for Stein's Unbiased Risk Estimate.
} 


\subsection{Generic PSF estimation}

We denote the function (or processing) of the observed data $\mathbf{y}$ by $\mathbf{F}(\mathbf{y})$. To estimate the PSF, instead of the standard MSE (mean squared error) given as $\frac{1}{N} \mathscr{E}\left\{\|\mathbf{F}(\mathbf{y})-\mathbf{x}\|^{2}\right\}[11,12]$, we consider the following blurred (filtered) version:

$$
\text { blur MSE }=\frac{1}{N} \mathscr{E}\left\{\left\|\mathbf{H F}(\mathbf{y})-\mathbf{H}_{0} \mathbf{x}\right\|^{2}\right\}
$$

as the objective functional to be minimized, where $\mathbf{H}$ and $\mathbf{H}_{0}$ are the estimated (tentative) and the unknown true PSF, respectively. Thus, we formulate the PSF estimation problem as the minimization of the blur MSE (3) over tentative $\mathbf{H}$.

We consider the processing $\mathbf{F}$ as the Wiener filtering, i.e.

$$
\mathbf{F}(\mathbf{y})=\underbrace{\left(\mathbf{H}^{\mathrm{T}} \mathbf{H}+\lambda \mathbf{I}\right)^{-1} \mathbf{H}^{\mathrm{T}}}_{\mathbf{W}_{\mathbf{H}, \lambda}} \mathbf{y}
$$

where $\lambda$ is the regularization parameter, $\mathbf{I}$ is identity matrix, $\mathbf{W}_{\mathbf{H}, \lambda}$ denotes the Wiener filtering with parameter $\lambda$. Given (4), the minimization of the blur MSE (3) yields the accurate estimated $\hat{\mathbf{H}}$ (i.e. very close to the true $\mathbf{H}_{0}$ ), if the value of $\lambda$ makes a good approximation of the true frequency-band indicator in Fourier domain [15], i.e.:

$$
\underbrace{\frac{\left|H_{0}(\omega)\right|^{2}}{\left|H_{0}(\omega)\right|^{2}+\sigma^{2} / S(\omega)}}_{\text {accurate band-indicator }} \approx \underbrace{\frac{|\hat{H}(\omega)|^{2}}{|\hat{H}(\omega)|^{2}+\lambda}}_{\text {approximated }} \text { for } \forall \omega
$$

where $H_{0}(\omega)$ and $\hat{H}(\omega)$ denote the frequency coefficients of true $\mathbf{H}_{0}$ and the estimated $\hat{\mathbf{H}}$, respectively, $S(\omega)$ is the power density spectrum of $\mathbf{x}$. Refer to [15] for the detailed justification of essential equivalence between the blur-MSE minimization (3) and approximation of the band-indicator (5). [15] also demonstrated the approximation (5) in Gaussian blur case. Fig. 2 in Section 2.2 will visualize the equivalence for the motion blur case.

Notice that we cannot directly minimize (3), as $\mathbf{H}_{0} \mathbf{x}$ is unknown in practice. However, [15] showed that the quantity of (3) can be replaced by a statistical unbiased estimate blur SURE, involving only the measurements $\mathbf{y}$, given as [15]:

$$
\tilde{\epsilon}_{\lambda}(\mathbf{H})=\frac{1}{N}\left\{\left\|\mathbf{H} \mathbf{W}_{\mathbf{H}, \lambda} \mathbf{y}-\mathbf{y}\right\|^{2}+2 \sigma^{2} \operatorname{Tr}\left(\mathbf{H} \mathbf{W}_{\mathbf{H}, \lambda}\right)\right\}-\sigma^{2}
$$

where $\mathbf{W}_{\mathbf{H}, \lambda}$ is given in (4), Tr denotes the matrix trace. Finally, we formulate the PSF estimation as the joint minimization of the blur SURE (6) over both $\mathbf{H}$ and $\lambda$. Fig. 1 summarizes the procedure of generic PSF estimation.

\subsection{Formulation of estimating motion blur parameters}

By taking motion blur for example, this section is to exemplify that by minimizing the blur SURE (6), the estimated $\hat{\lambda}$ yields both the good approximation of the band-indicator (5) and the accurate estimation of the blur parameters.

Recalling (2), we denote the motion-blur kernel with blur length $L$ and angle $\theta$ by $\mathbf{h}_{L, \theta}$, and the corresponding convolution matrix by $\mathbf{H}_{L, \theta}$. Thus, we formulate (6) as the following

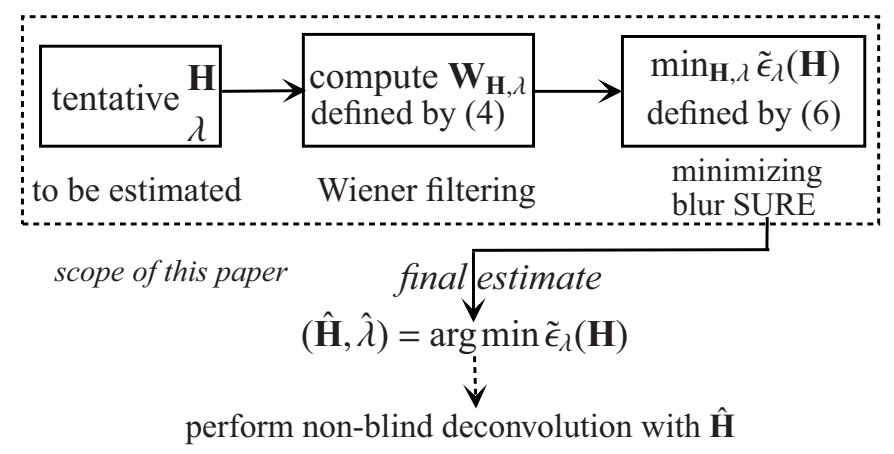

Fig. 1. The procedure of generic PSF estimation: joint minimization of blur SURE over $\mathbf{H}$ and $\lambda$, as shown in (6).

joint minimization problem:

$$
\begin{aligned}
\min _{L, \theta, \lambda} \tilde{\epsilon}_{\lambda}(L, \theta)=\min _{L, \theta, \lambda} & \frac{1}{N}\left\|\mathbf{H}_{L, \theta}\left(\mathbf{H}_{L, \theta}^{\mathrm{T}} \mathbf{H}_{L, \theta}+\lambda \mathbf{I}\right)^{-1} \mathbf{H}_{L, \theta}^{\mathrm{T}} \mathbf{y}-\mathbf{y}\right\|^{2} \\
& +\frac{2 \sigma^{2}}{N} \operatorname{Tr}\left(\mathbf{H}_{L, \theta}\left(\mathbf{H}_{L, \theta}^{\mathrm{T}} \mathbf{H}_{L, \theta}+\lambda \mathbf{I}\right)^{-1} \mathbf{H}_{L, \theta}^{\mathrm{T}}\right)-\sigma^{2}(7)
\end{aligned}
$$

over three decision variables: $L, \theta$ and $\lambda$.

We can use the alternating minimization to solve (7) between the three variables: to sequentially update one by fixing the other two, until the convergence is reached. The minimization over each variable can be efficiently performed by line search (see [15] for similar strategy).

Fig. 2 shows an example of minimizing (7), where the true motion blur kernel $\mathbf{h}_{L_{0}, \theta_{0}}$ is shown as (c). We can see that: (1) the estimated $\hat{\lambda}$ by minimizing (7) yields good approximation of band-indicator (shown in (a-2), (a-3), (a-4) and (d)); (2) with $\hat{\lambda}$, the blur-SURE minimization (7) can successfully find the accurate blur angle (shown in (a-1) - the curve of line search for $\theta$ ). Besides, Fig. 2-(a-1) also shows that blur SURE (6) is a good estimate of blur MSE (3).

\section{PRACTICAL IMPLEMENTATION FOR ESTIMATING MOTION BLUR PARAMETERS}

For motion blur case, we further experimentally obtain the following three observations. They may help us dramatically simplify the minimization problem (7).

Firstly, the accuracy of estimated $\hat{\theta}$ does not strongly depend on the value of $\lambda$. It means that for motion blur case, the minimization of blur SURE (7) is not so demanding in finding "optimal" $\lambda$. It is probably because that the band-indicator of (5) is prominently directional in Fourier domain. Thus, $\theta$, as the parameter describing the directionality, is much more dominant than the constant $\lambda$, since the essentially isotropic property of $\lambda$ does not show any directional preference. Consequently, the minimization of the blur SURE behaves more like finding $\theta$ to match the two directions of the strongly anisotropic band-indicators (i.e. both sides of (5)), rather than optimizing $\lambda$ to approximate (5) isotropically. 


$$
\text { “good" } \lambda_{1}=0.08
$$

(a-1) blur-SURE vs. $\theta$

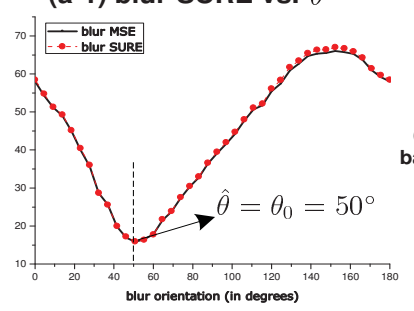

1-D presentation of band-indicator (a-3) blur direction

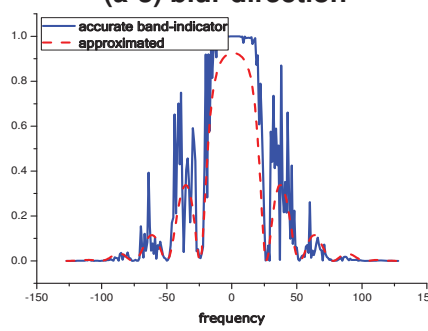

(c) true PSF(a-2) 2-D band-indicator

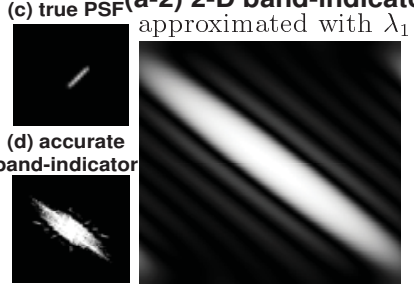

(a-4) perpendicular direction

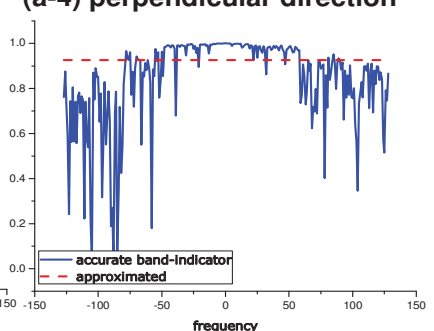

Fig. 2. Relation between blur-SURE minimization and approximation of band-indicator with estimated $\hat{\lambda}=0.08$. Example of Cameraman blurred by true $\mathbf{H}_{0}$ with $\theta_{0}=50^{\circ}$ (from horizontal line, shown in (c)) and $L_{0}=10$ pixels.

Fig. 3 shows an example to demonstrate the first claim. By comparing it with Fig. 2, we can see that using "bad" values of $\lambda$ (not obtained by (7)), the minimization of blur SURE still yields the accurate estimated $\hat{\theta}=\theta_{0}$, although the "bad" $\lambda_{2}$ makes a poor approximation of the band indicator (see Fig.3 (b-2), (b-3) and (b-4), compared to Fig. 2 (a-2), (a-3) and (a-4), respectively).

Secondly, it is highly preferred to estimate the blur direction first, and then, use the estimated $\hat{\theta}$ to estimate blur length. It is because that the blur SURE (6) is much more sensitive to $\theta$ than to $L$. The blur orientation is dominant in the motion blur: the minimization of blur SURE could accurately detect the angle $\theta$, even with incorrect tentative $L$ (i.e. quite different from true $L_{0}$ ). Fig. 4 shows an example of the minimization of the blur SURE with tentative $\hat{L}$ that is very different from true $L_{0}=21$ pixels. We can see that even with the two quite different tentative $\hat{L}=9$ or 45 pixels, the minimization of the blur SURE can still detect the accurate blur angle $\hat{\theta}=\theta_{0}=50^{\circ}$.

Finally, since the estimated $\hat{\theta}$ is highly accurate (compared to the ground-truth $\theta_{0}$ ), it is safe to find the blur length by minimizing the blur SURE with the accurate estimated $\hat{\theta}$. Experimentally, we found that $\lambda=2 \times 10^{-4} \sigma^{2}$ could well cope with various cases. Fig. 5 shows two examples.

Thus, summarizing the three observations, the minimization problem (7) can be simplified as the following Algorithm 1. Based on our second observation, the convergence speed of the alternating minimization is quite fast: generally, it reaches the convergence within only 3 steps.
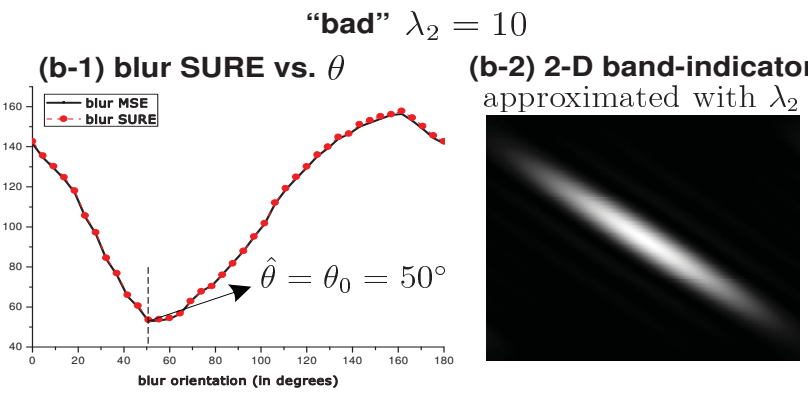

1-D presentation of band-indicator (b-3) blur direction

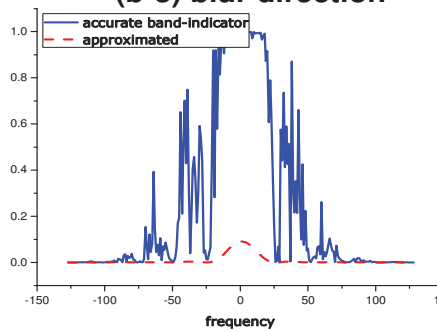

(b-4) perpendicular direction

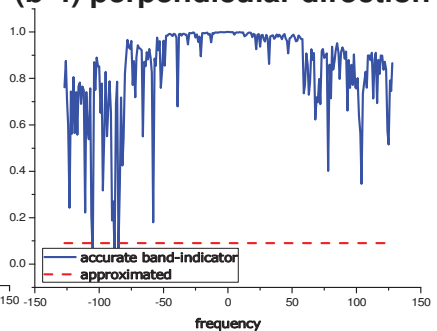

Fig. 3. Relation between blur-SURE minimization and approximation of band-indicator with non-optimal $\lambda_{2}=10$. Example of Cameraman blurred by true $\mathbf{H}_{0}$ with $\theta_{0}=50^{\circ}$ and $L_{0}=10$ pixels (shown in Fig. 2 (c)).

(a) $\hat{L}=9$ pixels and $\lambda=10$ (b) $\hat{L}=45$ pixels and $\lambda=10$
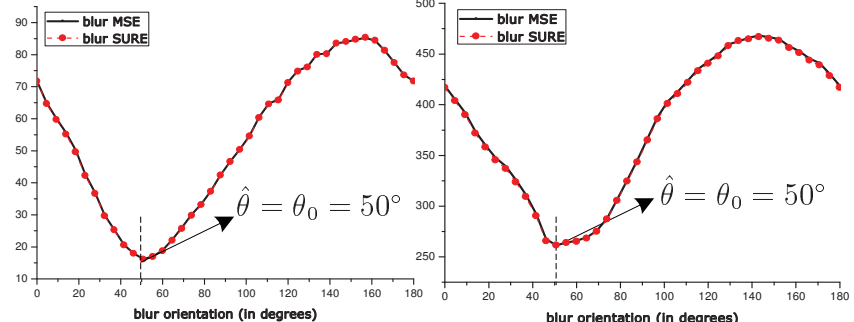

Fig. 4. The relation between the blur SURE (including blur MSE) and $\theta$, with incorrect tentative $\hat{L}$. Example of Cameraman blurred by true $\mathbf{H}_{0}$ with $\theta_{0}=50^{\circ}$ and $L_{0}=21$ pixels. (a) $L_{0}=7$ pixels and $\theta_{0}=90^{\circ}$

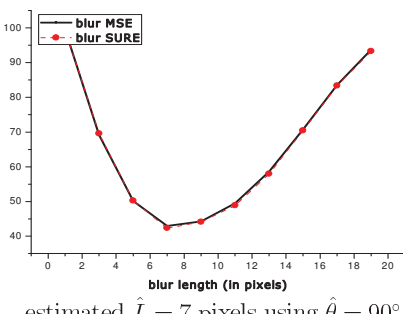

estimated $\hat{L}=7$ pixels using $\hat{\theta}=90^{\circ}$ (b) $L_{0}=33$ pixels and $\theta_{0}=45^{\circ}$

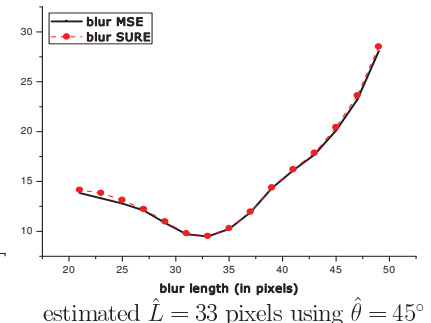

Fig. 5. The relation between the blur SURE (including blur MSE) and $L$, with $\hat{\theta}=\theta_{0}$ and $\lambda=2 \times 10^{-4} \sigma^{2}$. (image: Cameraman) 


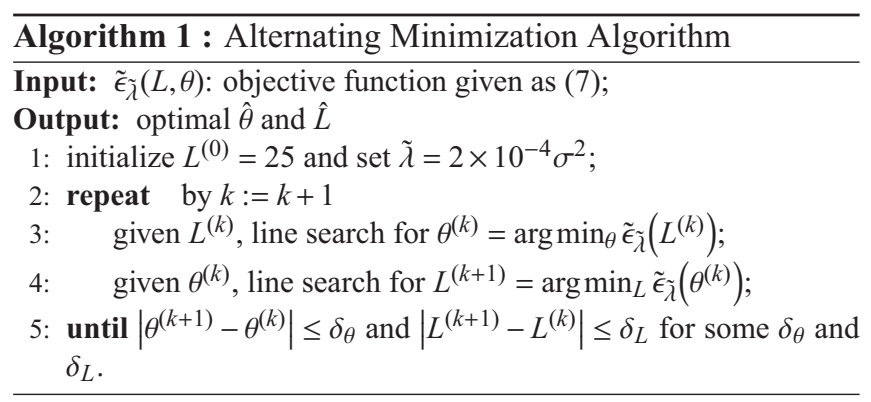

\section{EXPERIMENTAL RESULTS}

\subsection{Comparison with the state-of-the-art}

We perform the experiments on Mandrill image ${ }^{2}$, under $\mathrm{SNR}=10 \mathrm{~dB}$ [3]. The performance is evaluated by the absolute error $e_{\theta}$ (in degrees) and $e_{L}$ (in pixels), defined as [3]:

$$
e_{\theta}=\left|\theta_{0}-\hat{\theta}\right| ; \quad e_{L}=\left|L_{0}-\hat{L}\right|
$$

Tables 1-2 show the comparison of estimating blur angle and length, with Cepstral and Radon method [3]. We can observe that the proposed method outperforms the other methods in average.

Table 1. The error $e_{\theta}$ of estimated $\hat{\theta}$ (blur length $L_{0}=10$ )

\begin{tabular}{|c||c|c|c|c|c|c|}
\hline angle & $10^{\circ}$ & $40^{\circ}$ & $80^{\circ}$ & $100^{\circ}$ & $130^{\circ}$ & $170^{\circ}$ \\
\hline \hline Cepstral & $29^{\circ}$ & $17^{\circ}$ & $7^{\circ}$ & $9^{\circ}$ & $\mathbf{3}^{\circ}$ & $8^{\circ}$ \\
\hline Radon & $3^{\circ}$ & $12^{\circ}$ & $\mathbf{2}^{\circ}$ & $9^{\circ}$ & $23^{\circ}$ & $\mathbf{1}^{\circ}$ \\
\hline ours & $\mathbf{2}^{\circ}$ & $\mathbf{1}^{\circ}$ & $\mathbf{2}^{\circ}$ & $\mathbf{2}^{\circ}$ & $\mathbf{3}^{\circ}$ & $2^{\circ}$ \\
\hline
\end{tabular}

Table 2. The error $e_{L}$ of estimated $\hat{L}$

\begin{tabular}{|c||c|c|c|c|c|c|}
\hline length & 10 & 20 & 30 & 40 & 50 & 60 \\
\hline \hline 1-D Cepstral & 35 & 15 & 5 & 8 & 15 & 17 \\
\hline 2-D Cepstral & 10 & $\mathbf{2}$ & 1 & 1 & $\mathbf{2}$ & 7 \\
\hline ours & $\mathbf{5}$ & $\mathbf{2}$ & $\mathbf{0}$ & $\mathbf{0}$ & 3 & $\mathbf{6}$ \\
\hline
\end{tabular}

\subsection{Application to real motion-blurred image}

In our last set of experiments, the method is applied to a real motion-blurred image Pavilion captured by a digital camera, shown in Fig.6-(a). The estimated parameters of motion blur from Fig.6-(a) are $\hat{\theta}=0^{\circ}$ and $\hat{L}=15$ pixels, obtained by our approach. We use our developed SURE-LET approach to perform the non-blind deconvolution with the estimated blur kernel [14]. Fig.6-(b) shows the restored image.

\section{CONCLUSION}

In this paper, we proposed a new method to estimate motion blur parameters based on a new criterion - blur SURE: a statistical estimate of blur MSE. Both blur orientation and

\footnotetext{
${ }^{2}$ Mandrill is available at http://decsai.ugr.es/cvg/CG/base.htm (\#47)
}

(a) Observed image

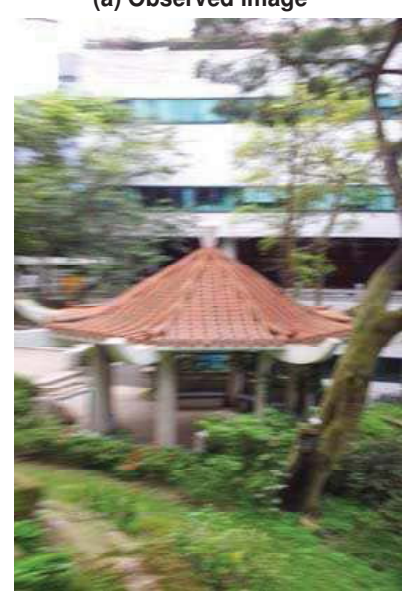

(b) Restored image

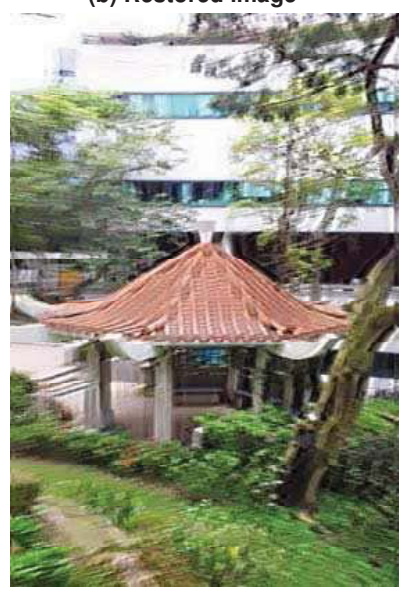

Fig. 6. Restoration of Pavilion: the estimated noise std is $\sigma=0.53$ by using MAD (median absolute deviation) [16].

length are accurately estimated by minimizing the blur SURE, incorporated with Wiener filtering.

Results obtained show that the proposed method has significant improvement of quality both numerically and visually. Compared to the other methods, the main advantage of our approach is that 1) the estimation of both blur angle and length is performed in a unified framework, instead of individually applying Radon and cepstral methods; 2) it does not need to analyze 2-D cepstrum and measure the prominent peaks [3-6], which is easily affected by the severe noise corruption.

It is worth noting that this paper is but an exemplification of SURE-type approach to motion blur estimation. SUREtype minimization itself does not specify any particular parametric form of PSF. There is huge potential to develop specific algorithms for various application, e.g. fluorescence microscopy [17], based on SURE-type minimization.

\section{ACKNOWLEDGMENT}

The authors would like to thank Mr. Tianxiang BAI for capturing the motion-blurred image Pavilion, shown in Fig.6-(a).

\section{REFERENCES}

[1] R. Fergus, B. Singh, A. Hertzmann, S.T. Roweis, and W.T. Freeman, "Removing camera shake from a single photograph," in ACM Transactions on Graphics (TOG). ACM, 2006, vol. 25, pp. 787-794.

[2] A. Levin, Y. Weiss, F. Durand, and W.T. Freeman, "Understanding and evaluating blind deconvolution algorithms," in Computer Vision and Pattern Recognition, 2009. CVPR 2009. IEEE Conference on. Ieee, 2009, pp. 1964-1971.

[3] F. Krahmer, Y. Lin, B. McAdoo, K. Ott, J. Wang, D. Widemann, and B. Wohlberg, "Blind image deconvolution: Motion blur estimation," IMA Preprints Series, pp. 2133-5, 2006. 
[4] M. Ebrahimi Moghaddam and M. Jamzad, "Motion blur identification in noisy images using mathematical models and statistical measures," Pattern recognition, vol. 40, no. 7, pp. 1946-1957, 2007.

[5] J. Oliveira, M. Figueiredo, and J. Bioucas-Dias, "Blind estimation of motion blur parameters for image deconvolution," Pattern Recognition and Image Analysis, pp. 604-611, 2007.

[6] S. Hongwei, M. Desvignes, and Y. Yunhui, "Motion blur adaptive identification from natural image model," in Image Processing (ICIP), 2009 16th IEEE International Conference on. IEEE, 2009, pp. 137-140.

[7] Q. Shan, J. Jia, and A. Agarwala, "High-quality motion deblurring from a single image," in ACM Transactions on Graphics (TOG). ACM, 2008, vol. 27 , p. 73.

[8] J. Biemond, R.L. Lagendijk, and R.M. Mersereau, "Iterative methods for image deblurring," Proceedings of the IEEE, vol. 78, no. 5, pp. 856-883, 1990.

[9] S.R. Deans, The Radon transform and some of its applications, John Wiley and Sons, 1983.

[10] W.T. Freeman, E.H. Adelson, Massachusetts Institute of Technology Media Laboratory. Vision, and Modeling Group, "The design and use of steerable filters," IEEE Transactions on Pattern analysis and machine intelligence, vol. 13, no. 9, pp. 891-906, 1991.

[11] T. Blu and F. Luisier, "The SURE-LET approach to image denoising," Image Processing, IEEE Transactions on, vol. 16, no. 11, pp. 27782786, 2007.

[12] Y.C. Eldar, "Generalized SURE for exponential families: Applications to regularization," Signal Processing, IEEE Transactions on, vol. 57, no. 2, pp. 471-481, 2009.

[13] J.C. Pesquet, A. Benazza-Benyahia, and C. Chaux, "A SURE approach for digital signal/image deconvolution problems," Signal Processing, IEEE Transactions on, vol. 57, no. 12, pp. 4616-4632, 2009.

[14] F. Xue, F. Luisier, and T. Blu, "SURE-LET image deconvolution using multiple wiener filters," Image Processing, 2012 IEEE International Conference on, submitted.

[15] F. Xue and T. Blu, "SURE-based blind Gaussian deconvolution," 2012 IEEE Statistical Signal Processing Workshop, submitted.

[16] D.L. Donoho and J.M. Johnstone, "Ideal spatial adaptation by wavelet shrinkage," Biometrika, vol. 81, no. 3, pp. 425-455, 1994.

[17] P. Pankajakshan, L. Blanc-Féraud, B. Zhang, Z. Kam, J.C. OlivoMarin, J. Zerubia, et al., "Parametric blind deconvolution for confocal laser scanning microscopy -proof of concept," 2008. 